\title{
Dança como uma intervenção para melhorar a mobilidade e o equilíbrio em idosos: uma revisão de literatura
}

\author{
Dance as an intervention to improve mobility and balance in \\ elderly: a literature review
}

Cléia Rocha de Oliveira (https://orcid.org/0000-0001-6891-8836) ${ }^{1}$

Anelise Ineu Figueiredo (https://orcid.org/0000-0002-7133-0432) ${ }^{1}$

Aniuska Schiavo (https://orcid.org/0000-0002-3356-0149) ${ }^{1}$

Lucas Athaydes Martins (https://orcid.org/0000-0002-8479-1158) ${ }^{1}$

Maria Eduarda Telles (https://orcid.org/0000-0002-3105-0604) ${ }^{1}$

Giovana Adamatti Rodrigues (https://orcid.org/0000-0003-3955-7984) ${ }^{1}$

Regis Gemerasca Mestriner (https://orcid.org/0000-0001-9837-1691) ${ }^{1}$
${ }^{1}$ Pontifícia Universidade Católica do Rio Grande do Sul. Av. Ipiranga, 6681, Prédio $81,7^{\circ}$ andar, sala 701. 90619-900 Porto Alegre RS Brasil. regis.mestriner@pucrs.br

\begin{abstract}
The aim of the present review was to identify whether dance protocols can benefit mobility and balance in elderly. A literature review was conducted in which 927 potentially relevant studies were identified (published in Portuguese, English, French, German, Spanish or Italian). There was no publication period restriction. After reading the titles, abstracts and review of the exclusion criteria, 15 randomized controlled trials were included. Most of the studies evaluated female subjects, using heterogeneous protocols of intervention and unspecific control groups. In addition, the period of exposure to dance was generally short: 2.6 weekly practices, of 59.1 minutes each, performed through 12.1 weeks. Dance was shown to be beneficial in $77.6 \%$ of the evaluated outcomes, exhibiting a moderate effect size for static balance and functional balance; and small effect size for mobility and strength/resistance of the lower limbs. However, future studies with the use of specific control groups and adoption of longer lasting protocols are necessary to evaluate the actual size effect that dance has on the maintenance of mobility and balance in elderly.
\end{abstract}

Key words Dance, Balance, Mobility, Elderly
Resumo O objetivo da presente revisão foi identificar se protocolos de dança podem beneficiar a mobilidade e o equilíbrio em idosos. Foi realizada uma revisão de literatura na qual identificou-se 927 estudos potencialmente relevantes, publicados em português, inglês, francês, alemão, espanhol ou italiano. Não houve restrição de período de publicação. Após a leitura dos títulos, resumos e revisão dos critérios de exclusão, 15 ensaios clínicos controlados e randomizados foram incluídos. A maioria dos estudos avaliaram pessoas do gênero feminino, com protocolos de intervenção heterogêneos e grupos controles pouco específicos. Além disso, o periodo de exposição à dança foi geralmente curto: aproximadamente 2,6 práticas semanais, de 59,1 minutos cada, realizadas ao longo de 12,1 semanas. A dança mostrou-se benéfica em 77,6\% dos desfechos avaliados, exibindo um tamanho de efeito moderado para o equilíbrio estático e equilíbrio funcional; embora pequeno para mobilidade e força/resistência dos membros inferiores. Contudo, estudos futuros com o uso de grupos controles específicos e adoção de protocolos mais duradouros são necessários para avaliar o real tamanho de efeito que a dança possui sobre a manutenção da mobilidade e equilíbrio em idosos.

Palavras-chave Dança, Equilíbrio, Mobilidade, Idosos 


\section{Introdução}

O envelhecimento humano é considerado um processo natural, contínuo e inexorável, no qual ocorrem alterações morfológicas, funcionais, químicas/neuroquímicas e psicológicas, que resultam na perda gradativa da vitalidade e da capacidade de adaptação do indivíduo ao meio ambiente. A depender da intensidade de tais alterações existirá maior ou menor susceptibilidade à incidência de doenças ${ }^{1-3}$. Neste contexto, a fragilidade física é um dos fatores que predispõem o sujeito às disfunções de funcionalidade e participação na vida diária. Sabe-se que esta tipicamente acompanha a perda de peso não intencional, redução da performance muscular (tanto aeróbia quanto anaeróbia), exaustão e baixa velocidade e/ou elevada variabilidade da marcha, dentre outros achados clínicos ${ }^{4-8}$. Ainda, as disfunções de mobilidade resultam no aumento do risco para a ocorrência de quedas da própria altura, contribuindo para um aumento da morbimortalidade em idosos. Cabe salientar que as quedas não resultam apenas em incapacidade física, mas frequentemente deflagram problemas psicológicos e sociais, prejudicando ainda mais a saúde desta população $0^{9-11}$. Ante ao exposto, a busca por estratégias que sejam capazes de contribuir para um envelhecimento bem-sucedido constitui-se como um importante desafio para a saúde pública mundial.

A dança é uma atividade física e social agradável, motivadora, que envolve a musicalidade e que pode ser desenvolvida de forma grupal ou individual. Tal atividade desafia o controle motor por meio de movimentos coreografados e realizados dentro de compassos musicais. Acreditase que o hábito de dançar possa prevenir alguns dos efeitos deletérios que levam à fragilidade no idoso, sendo capaz de estimular o sistema cognitivo e neuromusculoesquelético. Assim, a dança pode atuar de forma protetora na prevenção de quedas e na manutenção do equilíbrio corporal, contribuindo, desta maneira, para uma melhor qualidade de vida, funcionalidade e um envelhecimento mais saudável ${ }^{12-15}$. Culturalmente, a dança pode ser praticada durante todas as etapas da vida, podendo suscitar sentimentos positivos e sensação de bem-estar. Existem estudos na literatura sugerindo que esta prática também pode ser benéfica para melhorar a auto expressão, a comunicação, a motivação, a percepção de diversão e prazer com a vida, a espiritualidade, a identificação cultural, assim como promover uma ruptura e revitalização da sociedade ${ }^{15,16}$. No entanto, ainda não está suficientemente claro se tal prática pode ser benéfica para prevenir a ocorrência de quedas e proporcionar a manutenção do equilíbrio funcional em idosos, o que seria extremamente útil para a promoção e prevenção em saúde.

Dessa forma, o objetivo do presente estudo foi realizar uma revisão da literatura visando identificar se protocolos de intervenção utilizando a dança podem beneficiar a mobilidade e o equilíbrio postural em idosos.

\section{Métodos}

\section{Estratégia de busca}

Foi realizada uma revisão de literatura nas bases de dados PubMed e Cochrane Library e incluídos artigos publicados nos idiomas português, inglês, francês, alemão, espanhol ou italiano, sem restrição de ano de publicação. Para a busca, foram utilizados os seguintes descritores e seus termos relacionados: Dance Therapy, Dancing, Aged, 80 and over, aged. Além disso, utilizou-se o filtro randomized controlled trial $[p t]$ a fim de selecionar apenas ensaios clínicos randomizados (ECR). O plano metodológico desta revisão foi publicado na base de registros International prospective register of systematic reviews (PROSPERO) sob registro CRD42018093303, e está demonstrado no Quadro 1. Para a condução desta revisão foram seguidos critérios da Preferred Reporting Items for Systematic Reviews and Meta-Analyses (PRISMA Statement).

\section{Critérios de elegibilidade}

A presente revisão incluiu ECR que avaliassem intervenções terapêuticas de dança em idosos. Os critérios de inclusão foram: (1) participantes acima de 60 anos de idade submetidos a um protocolo de dança, (2) realização de testes que avaliassem mobilidade/equilíbrio dinâmico, equilíbrio estático e/ou força de membros inferiores. Foram excluídos artigos que não estivessem disponíveis para a leitura na íntegra.

\section{Seleção dos estudos e extração dos dados}

Os títulos e resumos dos artigos identificados nas buscas foram analisados, de forma independente, por 2 revisores e eventuais discordâncias encontradas no processo de seleção foram resolvidas por consenso. Os resumos que não apre- 
Quadro 1. Exemplo da estratégia de busca na base de dados Medline (Pubmed).

\section{PICOT (Problema, Intervenção, Comparação, Desfechos e Tipo de estudo)}

P: A dança pode beneficiar a mobilidade e o equilíbrio postural em idosos?

I: Qualquer tipo de dança.

C: Sedentário ou outro tipo de atividade física.

O: Mobilidade, equilíbrio estático e dinâmico.

T: Ensaio clínico controlado e randomizado.

Descritores e Termos relacionados

\#1 "Dance Therapy” OR “Therapy, Dance” OR "Dance Therapies” OR "Therapies, Dance”;

\#2 "Dancing” OR “Dance” OR "Ballet” OR “Square Dance” OR “Dance, Square” OR "Hip-Hop Dance” OR

"Dance, Hip-Hop" OR "Hip Hop Dance” OR “Jazz Dance” OR "Dance, Jazz” OR “Tap Dance” OR “Dance, Tap" OR “Modern Dance” OR “Dance, Modern” OR “Salsa Dancing” OR “Dancing, Salsa” OR “Line Dancing” OR "Dancing, Line";

\#3 “Aged, 80 and over" OR “Oldest Old" OR “Nonagenarians" OR “Nonagenarian” OR "Octogenarians" OR

"Octogenarian" OR “Centenarians" OR “Centenarian";

\#4 "Aged” OR "Elderly";

\#5 (randomized controlled trial[pt] OR controlled clinical trial[pt] OR randomized controlled trials[mh] OR random allocation $[\mathrm{mh}]$ OR double-blind method $[\mathrm{mh}]$ OR single-blind method $[\mathrm{mh}]$ OR clinical trial[pt] OR clinical trials $[\mathrm{mh}]$ OR (“clinical trial” $[\mathrm{tw}])$ OR $\left(\left(\operatorname{singl}^{\star}[\mathrm{tw}]\right.\right.$ OR doubl ${ }^{\star}[\mathrm{tw}]$ OR trebl${ }^{\star}[\mathrm{tw}]$ OR tripl$\left.{ }^{\star}[\mathrm{tw}]\right)$ AND $\left(\operatorname{mask}^{\star}[\mathrm{tw}]\right.$ OR blind $\left.{ }^{\star}[\mathrm{tw}]\right)$ ) OR (“latin square” $\left.[\mathrm{tw}]\right)$ OR placebos[mh] OR placebo* ${ }^{\star}[\mathrm{tw}]$ OR $\mathrm{random}^{\star}[\mathrm{tw}]$ OR research design [mh:noexp] OR follow-up studies[mh] OR prospective studies[mh] OR cross-over studies $[\mathrm{mh}]$ OR control${ }^{\star}[\mathrm{tw}]$ OR prospectiv*${ }^{\star}[\mathrm{tw}]$ OR volunteer $\left.{ }^{\star}[\mathrm{tw}]\right)$ NOT (animal[mh] NOT human $\left.[\mathrm{mh}]\right)$.

$\# 1$ OR \#2 = \#6;

$\# 3$ OR \#4 = \#;

Busca final = \#6 AND \#7 AND \#5.

sentaram a informação completa em relação aos critérios de elegibilidade foram selecionados para a leitura na íntegra. Os desfechos de interesse para o presente estudo foram mobilidade, equilíbrio estático e força nos membros inferiores. Além disso, foi utilizado um formulário para a extração de dados adicionais, tais como características da amostra, modalidade da dança utilizada, frequência, duração e o tempo de intervenção.

\section{Análise dos dados}

Os dados de interesse foram extraídos de cada um dos estudos incluídos pelos revisores independentes e expressos em média $(\mu)$, mediana $(M)$ e porcentagem (\%), bem como desvio padrão (DP), erro padrão (EPAD), intervalos interquartílicos 25\% (IQ25) e 75\% (IQ75). Além disso, como a realização de metanálise não foi aconselhada em decorrência da alta variabilidade metodológica dos estudos (vide a sessão de resultados para maiores detalhes) optou-se pelo cálculo do tamanho de efeito ( $\mathrm{d}$ de Cohen) intra e intergrupos para cada medida individual, sendo este pequeno entre 0,20 e 0,30 , médio entre 0,40 e 0,70 e grande quando $\geq 0,80$. Tal procedimento visou facilitar a leitura crítica conjunta dos resultados obtidos na revisão.

\section{Resultados}

Dentre os 927 artigos potencialmente relevantes, 15 deles foram selecionados de acordo com os critérios de elegibilidade. Todos os artigos incluídos foram escritos em língua inglesa e publicadas em periódicos indexados em bases de dados internacionais. Os detalhes relacionados ao processo de seleção dos artigos estão sumarizados na Figura 1. Em relação ao período de publicação, observou-se que os artigos incluídos foram publicados entre os anos de 1990 e 2016 . A qualidade metodológica dos estudos não foi avaliada empregando escalas específicas, contudo verificamos que a grande maioria dos trabalhos não apresentou detalhes suficientes para atingir níveis ótimos de qualidade metodológica conforme preconizado pela Cochrane Library.

O Quadro 2 apresenta o perfil das intervenções realizadas pelos grupos dança e as atividades utilizadas como controle nos estudos incluídos. Ainda no Quadro 2, observa-se que existiu uma 


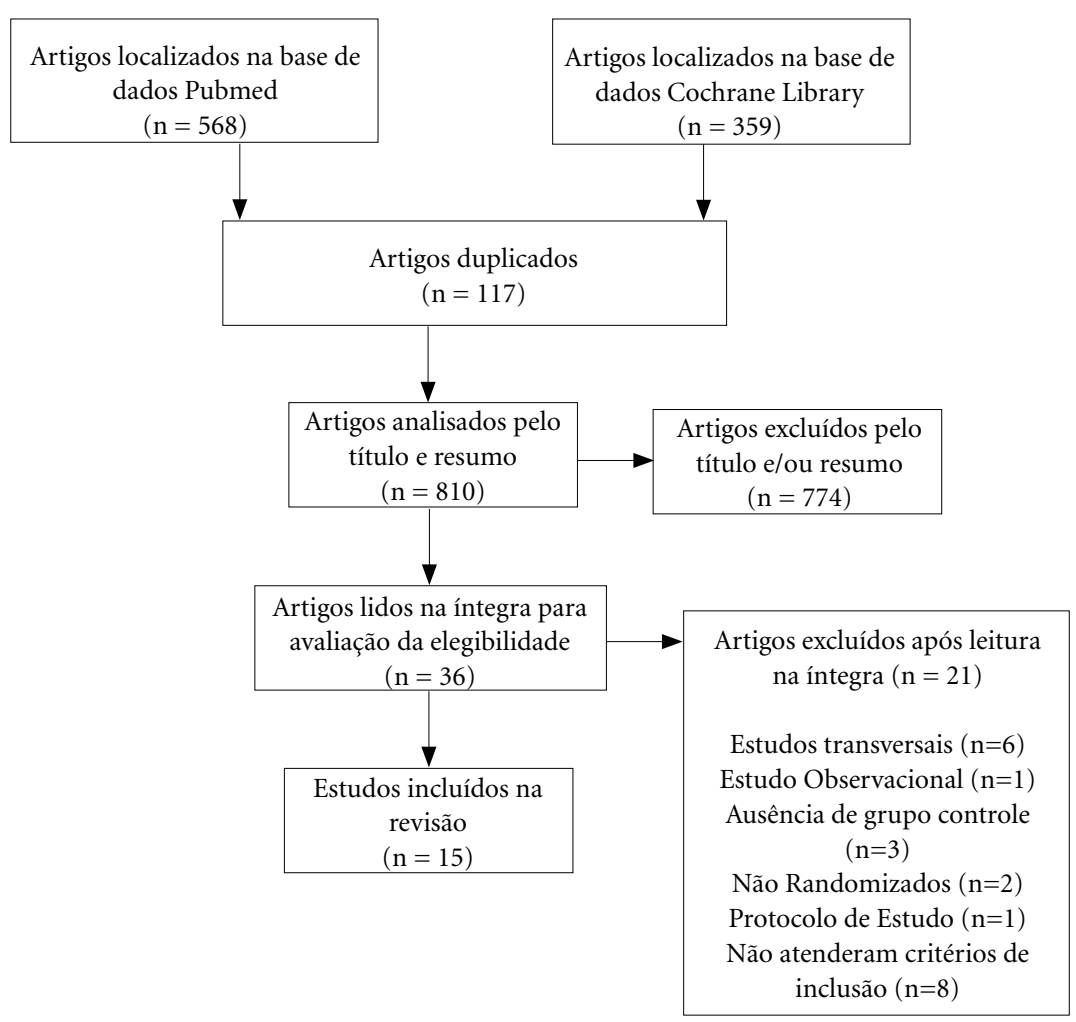

Figura 1. Fluxograma de revisão.

Quadro 2. Caracterização das modalidades de dança e as atividades comparadoras empregadas nos diferentes estudos.

\begin{tabular}{|c|c|c|}
\hline Estudo & Intervenção & Comparador \\
\hline Hopkins et al., $1990^{40}$ & Dança aeróbica de baixo impacto & Seguir atividades usuais/rotineiras \\
\hline Shigematsu et al., $2002^{41}$ & Dança baseada em exercícios aeróbicos & Seguir atividades usuais/rotineiras \\
\hline McKinley et al., $2008^{17}$ & Tango & Caminhadas \\
\hline Eyigor et al., $2009^{42}$ & $\begin{array}{l}\text { Dança folclórica turca e orientação sobre } \\
\text { caminhadas }\end{array}$ & Seguir atividades usuais/rotineiras \\
\hline Sofianidis et al., $2009^{20}$ & Dança tradicional grega & Não mencionado \\
\hline Borges et al., 2012 & Dança de salão & Seguir atividades usuais/rotineiras \\
\hline Granacher et al., $2012^{43}$ & Salsa & Seguir atividades usuais/rotineiras \\
\hline Janyacharoen et al., $2013^{18}$ & Dança tailandesa & $\begin{array}{l}\text { Orientações sobre exercícios aeróbicos } \\
\text { na rotina }\end{array}$ \\
\hline Kattenstroth et al., $2013^{21}$ & Dança para idosos (tipo não especificado) & Seguir atividades usuais/rotineiras \\
\hline Krampe, $2013^{23}$ & Dança aeróbica de baixo impacto & Seguir atividades usuais/rotineiras \\
\hline Borges et al., $2014^{44}$ & Dança de salão & Seguir atividades usuais/rotineiras \\
\hline Coubard et al., $2014^{45}$ & Dança contemporânea & Seguir atividades usuais/rotineiras \\
\hline Cruz-Ferreira et al., $2015^{46}$ & Dança criativa (sem estilo definido) & Seguir atividades usuais/rotineiras \\
\hline Eggenberger et al., $2016^{19}$ & Dança interativa com videogame & Treino de equilíbrio e alongamentos \\
\hline Merom et al., $2016^{37}$ & Dança folclórica australiana ou de salão* & Seguir atividades usuais/rotineiras \\
\hline
\end{tabular}

${ }^{\star}$ A escolha dependia da preferência do participante. 
grande variedade de protocolos de dança empregados, predominando as terminologias "dança de salão" (incluindo tango e salsa) e a "dança aeróbica de baixo impacto". Já em relação aos grupos controle, a grande maioria dos estudos empregou a manutenção das atividades usuais e rotineiras do idoso, sem o fornecimento de maiores detalhes metodológicos. Apenas três estudos utilizaram grupos controle com intervenção comparadora, sendo elas: a prática de caminhadas ${ }^{17}$, orientações sobre a implementação de exercícios aeróbicos na rotina ${ }^{18}$ e o treino de equilíbrio e alongamentos ${ }^{19}$. Um dos estudos não mencionou especificamente qual o tipo de intervenção controle utilizada ${ }^{20}$.

Em relação ao tamanho amostral, o número de sujeitos alocados no grupo dança variou entre 12 e 279 e, para o grupo controle, variou entre 10 e 251. Já em relação à idade dos idosos pesquisados, a média variou entre 64,9 e 85 anos. $\mathrm{O}$ gênero de maior prevalência foi o feminino.

Quanto ao tempo de intervenção, observouse grande variação nos estudos revisados (entre 4 e 48 semanas), com uma mediana igual a 12,1 semanas. A frequência semanal da prática das sessões/aulas de dança variou entre 1 e 3, com média de 2,6 sessões semanais. Apenas um estudo ${ }^{21}$ realizou 1 sessão semanal. $O$ tempo médio e o desvio padrão da duração de cada sessão/aula foi de 59 e 21,4 minutos, respectivamente. A Tabela 1 apresenta a compilação dos dados referentes às características dos volumes/doses de intervenção empregados.

Na Tabela 2 são apresentados os desfechos avaliados pelos diferentes estudos, antes e depois do período de intervenção, bem como o cálculo dos respectivos tamanhos de efeito intragrupo e intergrupos. $\mathrm{O}$ tamanho de efeito ( $\mathrm{d}$ de Cohen) foi considerado como pequeno $(0,20-0,30)$, médio $(0,40-0,70)$ ou grande $(\geq 0,80)$. Observase o predomínio do tamanho de efeito pequeno e médio para as análises intergrupos $(42,67 \%$ e $41,33 \%$, respectivamente), sendo que $16 \%$ dos desfechos avaliados tiveram tamanho de efeito grande.

Quanto às medidas relacionadas aos desfechos avaliados, observa-se que $59,9 \%$ delas estiveram relacionadas com o equilíbrio estático, $27,6 \%$ com a mobilidade/equilíbrio dinâmico, $9,2 \%$ com a força de membros inferiores e 9,2\% com escalas funcionais relacionadas ao equilíbrio. Cabe destacar, ainda, a existência de grande variabilidade metodológica nos procedimentos empregados para a mensuração dos desfechos de interesse, o que, somado ao baixo nível de rigor

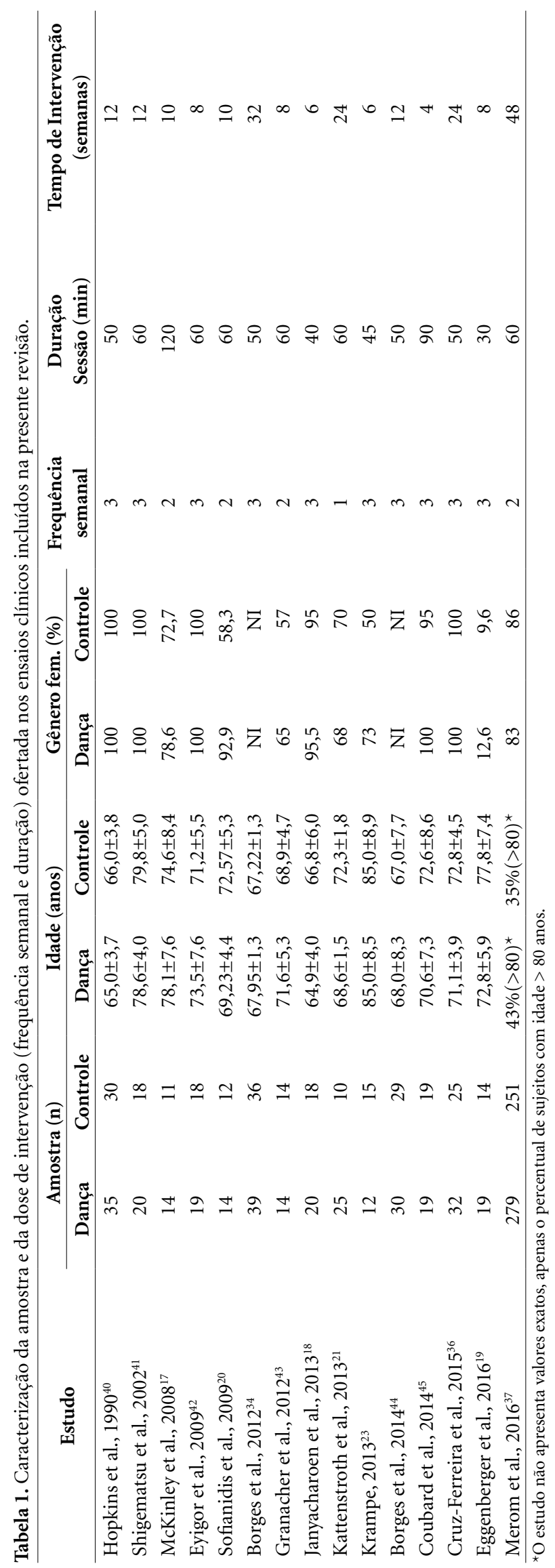




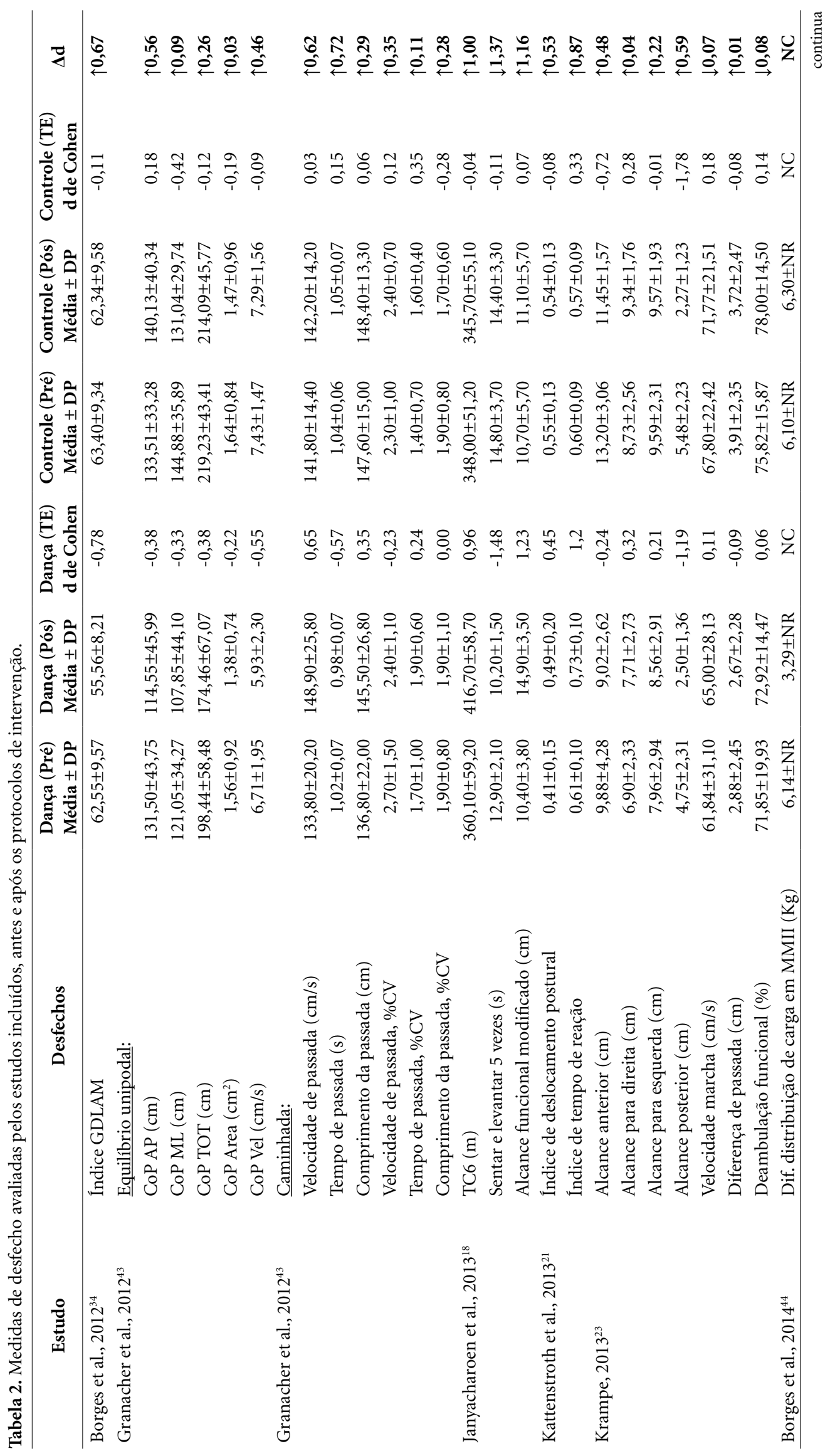




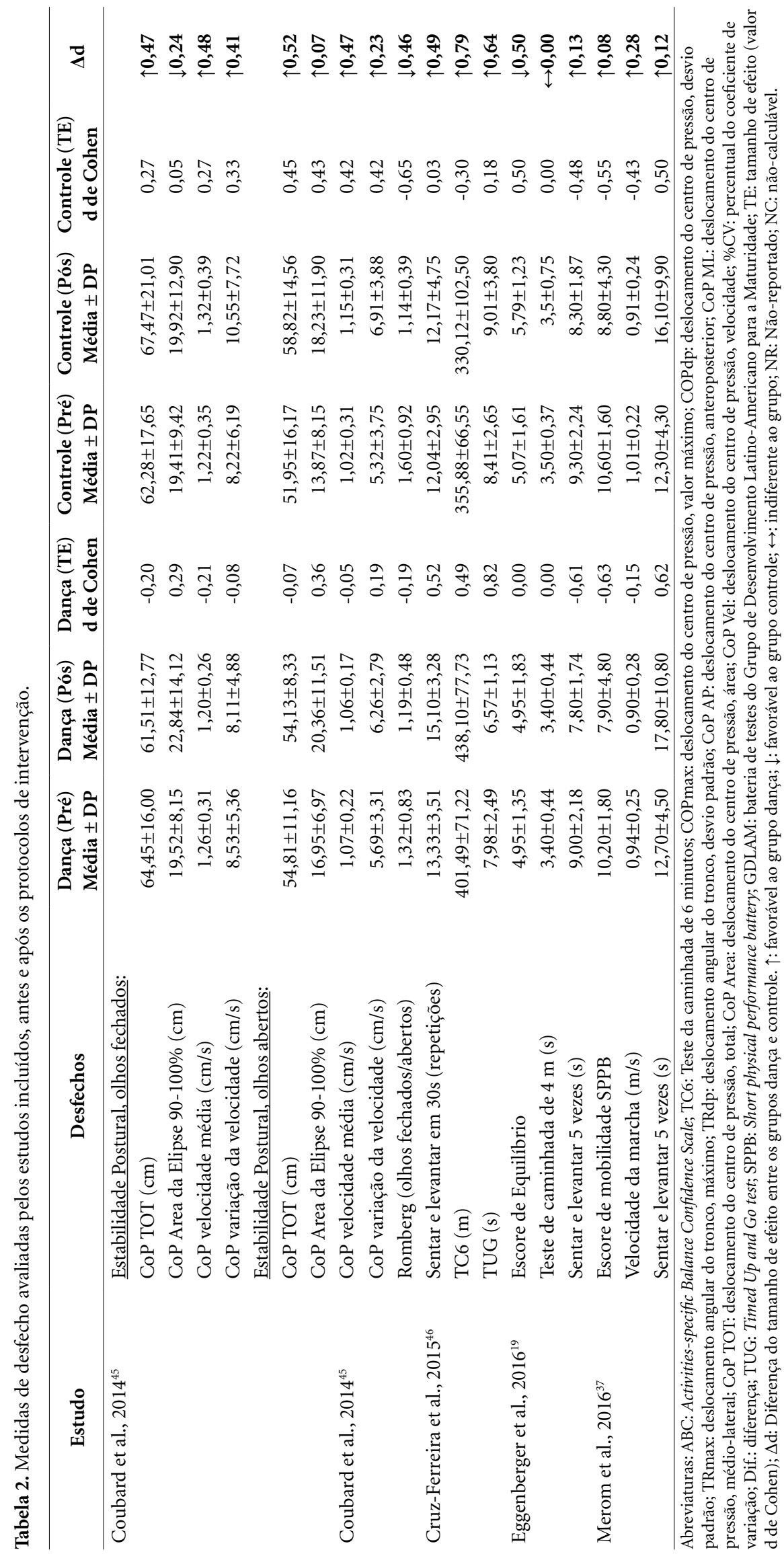


metodológico dos estudos incluídos e variabilidade nos protocolos de dança empregados, desaconselhou a realização de técnicas de metanálise na presente revisão. No entanto, para facilitar a leitura dos resultados, optamos por realizar uma descrição simplificada com base no percentual de efeitos benéficos conforme os tamanhos de efeito encontrados.

De um modo geral, 77,6\% das medidas realizadas pelos estudos sugeriram que a dança apresenta benefícios, enquanto $22,4 \%$ delas foram indiferentes ou favoráveis ao grupo controle. Especificamente em relação ao equilíbrio estático, $80,5 \%$ das medidas foram favoráveis à prática da dança, com mediana do tamanho de efeito igual a $0,42\left(\mathrm{IQ}_{25}=0,05 / \mathrm{IQ}_{75}=0,54\right)$. Por sua vez, as medidas de mobilidade/equilíbrio dinâmico foram favoráveis à dança em $76,2 \%$ dos casos, com mediana do tamanho de efeito igual a 0,28 $\left(\mathrm{IQ}_{25}=-0,07 / \mathrm{IQ}_{75}=0,64\right)$. Para a força de membros inferiores, as medidas também favoreceram a dança em $71,4 \%$ das avaliações, com mediana do tamanho de efeito igual a $0,13\left(\mathrm{IQ}_{25}=-0,14 /\right.$ $\left.\mathrm{IQ}_{75}=1,22\right)$. Por fim, os idosos expostos a dança também demonstraram resultados favoráveis nas escalas funcionais de equilíbrio em $85,7 \%$ das vezes, com mediana do tamanho de efeito igual a $0,56\left(\mathrm{IQ}_{25}=0,08 / \mathrm{IQ}_{75}=0,71\right)$.

\section{Discussão}

O presente estudo buscou observar se protocolos de intervenção utilizando a prática de dança são capazes de beneficiar a mobilidade e o equilíbrio postural em idosos, visto que o envelhecimento geralmente acarreta em mudanças fisiológicas e estruturais que impactam sobre o controle motor ${ }^{22}$.

De modo interessante, verificamos que 77,6\% das medidas de equilíbrio e mobilidade realizadas pelos estudos foram favoráveis à dança, predominando os tamanhos de efeito médios e pequenos, o que está de acordo com estudos prévios ${ }^{23}$. No entanto, observamos que a soma do percentual de tamanhos de efeito médios $(41,33 \%)$ e grandes $(16 \%)$ confere uma superioridade da prática de dança em $57,33 \%$ dos casos, o que é um dado bastante razoável. Recentemente, um estudo publicado por Fong Yan et al..$^{24}$ demonstrou que a prática de um programa de dança estruturado pode produzir efeitos semelhantes ou até mesmo superiores em comparação com outras atividades físicas, sendo a dança uma intervenção recomendável para o público idoso. Na mesma direção, Kattenstroth et al. ${ }^{22}$ sugerem que a prá- tica de dança possui bom potencial para promover benefícios relacionados à mobilidade e equilíbrio em idosos. Sabe-se que a manutenção de uma vida fisicamente ativa melhora a destreza de movimentos, reduz o risco de lesões e quedas da própria altura. Sendo assim, os efeitos da dança possivelmente estão associados com uma melhora do equilíbrio, da força e resistência muscular, da coordenação, ritmo, lateralidade e consciência corporal, contribuindo para a redução da ocorrência de quedas.

Apesar da análise geral dos resultados ser favorável aos sujeitos que praticam a dança quanto aos desfechos estudados, observa-se uma variabilidade muito grande entre os tamanhos de efeito. Assim, diversos fatores limitantes devem ser considerados quando da eleição da dança como atividade física de escolha. Tais fatores, inclusive, devem ser considerados para a realização de estudos futuros que sejam mais consistentes.

Inicialmente destaca-se o estilo de dança, uma vez que o estilo adotado poderia impactar na avaliação dos desfechos - haja vista que algumas danças são folclóricas enquanto outras são classificadas como danças de salão, dança criativa, ou até mesmo dança aeróbica, sem o fornecimento de maiores detalhes sobre as execuções realizadas. Ritmos e intensidades diferentes talvez produzam tamanhos de efeito diferentes, uma vez que uma dança lenta e com posturas estáticas tende a estimular o sistema neuromusculoesquelético de modo diferente de danças aeróbicas, de maior explosão ou, ainda, daquelas que possuem um maior componente de instabilidade ${ }^{25}$.

Um estudo recente sugere que dançarinos profissionais apresentam maior capacidade do sistema postural em gerenciar a instabilidade, sendo o feedback visual um dos principais componentes neste process ${ }^{26}$. Portanto, o grau de proficiência e experiência com a prática de dança também deve ser considerada quando avaliamos os efeitos de tal intervenção.

Outro aspecto potencialmente relevante para a variabilidade dos tamanhos de efeito observados diz respeito a escolha da intervenção comparadora (grupo controle). Na presente revisão, observamos que a grande maioria dos estudos utilizam grupos controle inespecíficos. Assim, pode-se concluir que a prática de dança é superior a manutenção da rotina usual, supostamente sedentária, mas não se pode concluir que a dança será realmente superior à outras formas de atividade física. Além disso, um estilo de vida sedentário, sabidamente ligado a disfunções fisiológicas e doenças crônicas, pode representar, 
potencialmente, um viés sobre determinadas interpretações de um dado estudo, o que deve ser considerado ao analisarmos os seus resultados ${ }^{27}$.

Evidencia-se também que os estudos não descrevem suficientemente como ocorre a estruturação dos protocolos de dança empregados, o que possivelmente impacta nos volumes de treino e doses efetivamente entregues. Sabe-se que volumes e estruturas de treino pouco definidos dificultam a obtenção dos resultados desejados ${ }^{28}$, bem como a avaliação da reprodutibilidade dos mesmos $^{29}$.

Em relação ao tempo de intervenção, observase que a maioria dos estudos empregou protocolos relativamente curtos, cerca de 3 meses. Embora frequentemente adotado de forma protocolar em estudos com intervenções baseadas em movimento, estas típicas 12 semanas não necessariamente são suficientes para estimar um tamanho de efeito máximo da intervenção se considerarmos os princípios fisiológicos do exercício ${ }^{30}$. Sendo assim, novos estudos mais longos, com acurado controle de volumes/doses efetivamente entregues por sessão de prática, são necessários para avaliar o real tamanho de proteção que a dança possui sobre o equilíbrio e mobilidade de pessoas idosas.

É relevante ressaltar que os procedimentos/ métodos de avaliação utilizados variam consideravelmente entre os estudos, o que dificulta a precisão na comparabilidade entre os achados e a interpretação mais detalhada dos fenômenos observados em conjunto. Apesar disso, nós procuramos categorizar os testes empregados de acordo com seus desfechos relacionados para facilitar a leitura crítica da revisão. Assim, observou-se que $59,9 \%$ das medidas foram relacionadas com o equilíbrio estático. Portanto, não podemos afirmar que os tamanhos de efeito gerais encontrados com a prática de dança são aplicáveis igualmente para a mobilidade ${ }^{31}$ e/ou força e resistência de membros inferiores ${ }^{32}$ - variáveis físicas tipicamente associadas com a chance ou risco de quedas. Ademais, Britten e colaboradores ${ }^{33}$ relatam que a dança tem o potencial não só de minimizar o risco de quedas diretamente pela melhora nos componentes físicos, mas também atua no psicológico, como no medo de cair, no humor, na depressão, trazendo benefícios em mais de uma área da vida - variáveis estas que também compõem o espectro de complexidade que deve ser levado em consideração nos estudos que avaliam os efeitos da prática de dança.

Cabe ainda destacar que, dentre os vieses encontrados, está a insuficiente descrição da história de saúde pregressa e atual dos participantes dos estudos. Por exemplo, problemas relacionados propriocepção, audição ou visão podem ser, por si só, limitantes da performance do equilíbrio e da mobilidade, influenciando no tamanho de efeito da intervenção $0^{34-36}$.

Por fim, excetuando-se o estudo de Merom et al. ${ }^{37}$, que contou com 279 participantes, todos os demais possuem tamanho amostral mínimo ou insuficiente, dificultando a validação externa de seus achados. Ainda sobre as amostras estudadas, vale ressaltar que a grande maioria dos sujeitos era do gênero feminino. Isso pode dever-se a diversos fatores, tais como a dança ser historicamente atrelada como uma atividade feminina ${ }^{38}$ em decorrência da construção de estereótipos que setorizam atividades de acordo com o gênero. Outras possibilidades de especulação são a maior longevidade e maior costume de cuidar da saúde ${ }^{39}$, bem como a maior inscrição feminina em atividades desta natureza.

Conclui-se, desta maneira, que a prática de dança é capaz de promover efeitos protetores sobre o equilíbrio estático e funcional em idosos, embora seus efeitos possam ser superestimados pela carência de grupos controles mais específicos. Assim, novos estudos são necessários para determinar os reais efeitos de cada modalidade de dança e de suas doses ideais para a manutenção da mobilidade e equilíbrio em idosos.

\section{Colaboradores}

Todos os autores contribuíram para a formulação do artigo em questão e leram sua versão final. 


\section{Referências}

1. Arruda IEA. Reflexões sobre o idoso e o programa Universidade da Terceira Idade. Rev Bras Cien Envelhecimento Hum 2007; 4(2):98-99.

2. Barbosa NMC, Hunger DACF, Pereira VAO. Brincar em diferentes gerações: compartilhando experiências e atividades lúdicas na prática educativa. Rev Bras Cienc Envelhecimento Hum 2007; 4(2):127-135.

3. Fernandez-Arguelles EL, Rodriguez-Mansilla J, Antunez LE, Garrido-Ardila EM, Muñoz RP. Effects of dancing on the risk of falling related factors of healthy older adults: a systematic review. Arch Gerontol Geria$\operatorname{tr} 2015 ; 60(1): 1-8$.

4. Xue QL, Bandeen-Roche K, Varadhan R, Zhou J, Fried LP. Initial manifestations of frailty criteria and the development of frailty phenotype in the Women's Health and Aging Study II. J Gerontol A Biol Sci Med Sci 2008; 63(9):984-990.

5. Brasil. Ministério da Saúde (MS). Secretaria de Atenção à Saúde. Departamento de Atenção Básica. Envelhecimento e saúde da pessoa idosa. Brasília: MS; 2006. (Caderno de Atenção Básica no 19).

6. Keogh JW, Kilding A, Pidgeon P, Ashley L, Gillis D. Physical benefits of dancing for healthy older adults: a review. J Aging Phys Act 2009; 17(4):479-500.

7. Hayashida I, Tanimoto Y, Takahashi Y, Kusabiraki T, Tamaki J. Correlation between muscle strength and muscle mass, and their association with walking speed, in community-dwelling elderly Japanese individuals. PLoS One 2014; 9(11):1-6.

8. Song S, Geyer H. Predictive neuromechanical simulations indicate why walking performance declines with aging. J Physio 2018; 96(7):1199-1210.

9. Duarte M, Freitas SMSF. Revisão sobre posturografia baseada em plataforma de força para avaliação do equilíbrio. Rev Bras Fisioter 2010; 14(3):183-192.

10. Mahoney JR, Oh-Park M, Ayers E, Verghese J. Quantitative trunk sway and prediction of incident falls in older adults. Gait Posture 2017; 58:183-187.

11. Francis-Coad J, Etherton-Beer C, Bulsara C, Blackburn N, Chivers P, Hill AM. Evaluating the impact of a falls prevention community of practice in a residential aged care setting: a realist approach. $B M C$ Health Serv Res 2018; 18(1):21.

12. Nóbrega ACL, Freitas EV, Oliveira MAB, Leitão MB, Lazzoli JK, Nahas RM, Baptista CAS, Drummond FA, Rezende L, Pereira J, Pinto M, Radominski RB, Leite N, Thiele ES, Hernandes AJ, Araújo CGS, Teixeira JAC, Carvalho T, Borges SF, De Rose EH. Posicionamento Oficial da Sociedade Brasileira de Medicina do Esporte e da Sociedade Brasileira de Geriatria e Gerontologia: Atividade Física e Saúde no Idoso. Rev Bras Med Esporte 1999; 5(6):207-211.

13. Rehfeld K, Hökelmann A, Lehmann W, Blaser P. Auswirkungeneiner Tanz- und Kraft-Ausdauer-Intervention auf kognitive Fähigkeiten älterer Menschen. $Z$ Neuropsychol 2014; 25(2):99-108.
14. Rehfeld K, Müller P, Aye N, Schmicker M, Dordevic M, Kaufmann J, Hökelmann A, Müller NG. Dancing or fitness sport? The effects of two training programs on hippocampal plasticity and balance abilities in healthy seniors. Front Hum Neurosci 2017; 11:305.

15. Alpert PT, Miller S K, Wallmann H, Havey R, Cross C, Chevalia T, Gillis CB, Kodandapari K. The effect of modified jazz dance on balance, cognition, and mood in older adults. J Am Assoc Nurse Pract 2009; 21(2):108-115

16. Robatto L. Dança em processo, a linguagem do indizível. Salvador: Centro Editorial e Didático da UFBA; 1994.

17. McKinley P, Jacobson A, Leroux A, Bednarczyk V, Rossignol M, Fung J. Effect of a community-based Argentine tango dance program on functional balance and confidence in older adults. J Aging Phys Act 2008; 16(4):435-453.

18. Janyacharoen T, Laophosri M, Kanpittaya J, Auvichayapat P, Sawanyawisuth K. Physical performance in recently aged adults after 6 weeks traditional Thai dance: a randomized controlled trial. Clin Interv Aging 2013; 8:855-859.

19. Eggenberger P, Wolf M, Schumann M, De Bruin ED. Exergame and balance training modulate prefrontal brain activity during walking and enhance executive function in older adults. Front Aging Neurosci 2016; 8:66.

20. Sofianidis G, Hatzitaki V, Douka S, Grouios G. Effect of a 10-week traditional dance program on static and dynamic balance control in elderly adults. J Aging Phys Act 2009; 17(2):167-180.

21. Kattenstroth JC, Kalisch T, Holt S, Tegenthoff M, Dinse HR. Six months of dance intervention enhances postural, sensorimotor, and cognitive performance in elderly without affecting cardio-respiratory functions. Front Aging Neurosci 2013; 5:5.

22. Kattenstroth JC, Kolankowska I, Kalisch T, Dinse HR. Superior sensory, motor, and cognitive performance in elderly individuals with multi-year dancing activities. Front Aging Neurosci 2010; 2:31.

23. Krampe J. Exploring the effects of dance-based therapy on balance and mobility in older adults. West $J$ Nurs Res 2013; 35(1):39-56.

24. Fong Yan A, Cobley S, Chan C, Pappas E, Nicholson LL, Ward RE, Murdoch RE, Gu Y, Trevor BL, Vassallo AJ, Wewege MA, Hiller CE. The Effectiveness of Dance Interventions on Physical Health Outcomes Compared to Other Forms of Physical Activity: A Systematic Review and Meta-Analysis. Sports Med 2018; 48(4):933-951.

25. Golomer EM, Gravenhorst RM, Toussaint Y. Influence of vision and motor imagery styles on equilibrium control during whole-body rotations. Somatosens Mot Res 2009; 26(4):105-110. 
26. Michalska J, Kamieniarz A, Fredyk A, Bacik B, Juras G, Słomka KJ. Effect of expertise in ballet dance on static and functional balance. Gait Posture 2018; 1(64):6874.

27. Buford TW, Manini TM. Sedentary individuals as "controls" in human studies: the correct approach? Proc Natl Acad Sci USA 2010; 107(34):E134.

28. Mangine GT, Hoffman JR, Gonzalez AM, Townsend JR, Wells AJ, Jajtner AR, Beyer KS, Boone $\mathrm{CH}$, Miramonti AA, Wang R, La Monica MB, Fukuda DH, Ratamess NA, Stout JR. The effect of training volume and intensity on improvements in muscular strength and size in resistance-trained men. Physiol Rep 2015; 3(8):e12472.

29. Van Aert RCM, Van Assen MALM. Examining reproducibility in psychology: A hybrid method for combining a statistically significant original study and a replication. Behav Res Methods 2018; 50(4):1515-1539.

30. Rivera-Brown AM, Frontera WR. Principles of exercise physiology: responses to acute exercise and longterm adaptations to training. PMR 2012; 4(11):797804.

31. Suttanon P, Hill KD, Said CM, Dodd KJ. A longitudinal study of change in falls risk and balance and mobility in healthy older people and people with Alzheimer disease. Am J Phys Med Rehabil 2013; 92(8):676-685.

32. Wu F, Callisaya M, Wills K, Laslett LL, Jones G, Winzenberg T. Both Baseline and Change in Lower Limb Muscle Strength in Younger Women Are Independent Predictors of Balance in Middle Age: A 12-Year Population-Based Prospective Study. J Bone Miner Res 2017; 32(6):1201-1208.

33. Britten L, Addington C, Astill S. Dancing in time: feasibility and acceptability of a contemporary dance programme to modify risk factors for falling in community dwelling older adults. BMC Geriatr 2017; 17(1):83.

34. Borges EG, Cader SA, Vale RG, Cruz TH, Carvalho MC, Pinto FM, Dantas EH. The effect of ballroom dance on balance and functional autonomy among the isolated elderly. Arch Gerontol Geriatr 2012; 55(2):492-496.

35. Santos SR, Santos IBC, Fernandes MGM, Henriques MERM. Elderly quality of life in the community: application of the Flanagan's Scale. Rev Lat Am Enferm 2002; 10(6):757-764.

36. Perracini MR, Ramos LR. Fall-related factors in a cohort of elderly community residents. Rev Saude Publica 2002; 36(6):709-716.

37. Merom D, Grunseit A, Eramudugolla R, Jefferis B, Mcneill J, Anstey KJ. Cognitive benefits of social dancing and walking in old age: the Dancing Mind randomized controlled trial. Front Aging Neurosci 2016; 8:26.

38. Assis MDP, Saraiva MC. O feminino e o masculino na dança: das origens do balé à contemporaneidade. Movimento 2013; 19(2):303-323.
39. Camargos MCS, Gonzaga MR. Viver mais e melhor? Estimativas de expectativa de vida saudável para a população brasileira. Cad Saude Publica 2015; 31(7):1460-1472.

40. Hopkins DR, Murrah B, Hoeger WW, Rhodes RC. Effect of low-impact aerobic dance on the functional fitness of elderly women. Gerontologist 1990; 30(2):189192.

41. Shigematsu R, Chang M, Yabushita N, Sakai T, Nakagaichi M, Nho H, Tanaka K. Dance-based aerobic exercise may improve indices of falling risk in older women. Age Ageing 2002; 31(4):261-266.

42. Eyigor S, Karapolat H, Durmaz B, Ibisoglu U, Cakir S. A randomized controlled trial of Turkish folklore dance on the physical performance, balance, depression and quality of life in older women. Arch Gerontol Geriatr 2009; 48(1):84-88.

43. Granacher U, Muehlbauer T, Bridenbaugh S, Wolf M, Roth R, Gschwind YJ, Wolf I, Mata R, Kressig RW. Effects of a salsa dance training on balance and strength performance in older adults. Gerontology 2012; 58(4):305-312.

44. Borges EG, Vale RG, Cader SA, Leal S, Miguel F, Pernambuco CS, Dantas EH. Postural balance and falls in elderly nursing home residents enrolled in a ballroom dancing program. Arch Gerontol Geriatr 2014; 59(2):312-316

45. Coubard OA, Ferrufino L, Nonaka T, Zelada O, Bril B, Dietrich G. One month of contemporary dance modulates fractal posture in aging. Front Aging Neurosci 2014; 6:17.

46. Cruz-Ferreira A, Marmeleira J, Formigo A, Gomes D, Fernandes J. Creative Dance Improves Physical Fitness and Life Satisfaction in Older Women. Res Aging 2015; 37(8):837-855.

Artigo apresentado em 15/07/2018

Aprovado em 04/09/2018

Versão final apresentada em 06/09/2018 